\title{
Comprehensive Approach of Hybrid Nanoplatforms in Drug Delivery and Theranostics to Combat Cancer
}

\author{
Nanasaheb D. Thorat ${ }^{\mathrm{a}, \mathrm{b}}$, Helen Townley ${ }^{\mathrm{c}}$, Rakesh M Patil ${ }^{\mathrm{d}}$, Syed A.M.Tofail ${ }^{\mathrm{b}}$ and Joanna Bauer ${ }^{\mathrm{a}}$ \\ ${ }^{\text {a }}$ Department of Biomedical Engineering, Wroclaw University of Science and Technology, Wroclaw, Poland \\ ${ }^{b}$ Modelling Simulation and Innovative characterisation (MOSAIC), Bernal Institute, University of Limerick, \\ Ireland \\ 'Nuffield Department of Women's \& Reproductive Health and Department of Engineering Science, University \\ of Oxford, UK \\ ${ }^{\mathrm{d}}$ Directorate of Forensic Science Laboratories, Kalina, Santacruz (E), Mumbai, Maharashtra, India
}

Corresponding Author

Nanasaheb D. Thorat

Department of Biomedical Engineering,

Wroclaw University of Science and Technology,

Wroclaw, Poland

EMAIL: thoratnd@gmail.com, nanasaheb.thorat@pwr.edu.pl 


\begin{abstract}
To date, various chemically and biosynthesized nanoparticles, or hybrid nano systems, have been developed under the umbrella of nanomedicine. These may be introduced into the body either orally, nasally, intratumourally or intravenously. Successfully translating hybrid nanoplatforms from preclinical proof-of-concept to therapeutic value in the clinic is challenging. Having made significant advances with drug-delivery technologies, we must learn from other areas of oncology drug development, where patient stratification and target-driven design have improved patient outcomes. This review aims to identify gaps in our understanding of the current strengths of nanomedicine platforms in drug delivery and cancer theranostics. We report on the current approaches of nanomedicine at preclinical and clinical stages.
\end{abstract}

\title{
1. Introduction
}


The heterogeneous nature of cancerous tumors makes it challenging to present an accurate diagnosis and therefore efficient treatment. The interpatient tumor heterogeneity arises due to a high diversity of cancer sub-types, unique genetics and epigenetics, and dynamic factors such as age, environment, lifestyle, and medical history[1]. Thus, for the development and validation of effective treatments patient stratification needs to be optimal. However, conventional cancer therapeutic modalities such as chemotherapy and radiotherapy are lacking the patient-specific individualized platforms to combat cancer effectively. Suchindividualized platforms include the delivery of chemotherapeutic drugs in patient-specific clinical situations.

The use of nanotechnology in cancer medicine has the potential to have a major impact on diagnosis and treatment of tumors. Onegoal of nanomedicine is to develop therapeutic or diagnostic platforms using specially designed chemotherapeutic drug conjugated nanomaterials such as hybrid nanostructures (HNCs) to address cancer theranostics (therapy + diagnostics) [2-6]. Nanomedicine allows the navigation of HNCs through highly complex physiological networks and thus maximizes the delivery of chemotherapeutics to the tumor. HNCs have extraordinary surface functionality and such structures can enhance anticancer activity by boosting the triggered drug release directly at the tumor site[7-9]. By taking advantage of nanosize structures and chemotherapeutics, hybrid nanoplatforms may be promising for cancer theranostics because: (i) in addition to existing active/passive targeting processes, HNCs exhibit efficient accumulation and retention at tumor sites; (ii) HNCs have high penetration ability comparable to that of small molecules in the tumor region; (iii) HNCs carry and release chemotherapeutic drugs in response to internal and external triggers/stimuli to induce effective therapeutics, and (iv) pharmacokinetics of HNCs are similar to that of drugs or small molecules[10].

This review summarizes the diversity of hybrid nanoplatforms currently in use, underlying their recent applications in cancer theranostics.

\section{Hybrid nanoplatforms advancing cancer theranostics}

An ideal nanocarrier for cancer theranostics should simultaneously attain high tumor accumulation and an enhanced therapeutic effect[11]. In vivo, the designed nanocarriers should be able to change their functionality in different biological environments[12]. Stimuli-responsive materials, which are based on hybrid platforms, promise to achieve this goal. A hybrid nanoplatform (HNP) presumes the nanocarriers to show multifunctionality and to respond to stimuli either from within the tumor internal, or externally. They will also need to hae good 
stability in the blood circulatory system to achieve efficient passive targeting. The ability of hybrid nanoplatforms (HNPs) to carry multiple therapeutic agents increases their aptitude to improve cancer treatment. HNP based cancer theranostics can combine both the ability to diagnose and treat cancers. This non-conventional cancer therapy approach allows both monitoring of drug release and visualization of drug released in tumor. These properties show the potential of HNPs for use in personalized treatment $[13,14]$. New generation HNPs possess dual-targeted delivery of therapeutic molecules and therapeutic energy (such as heat).

Despite recent advances in HNPs to amplify therapeutic efficiency, these nanostructures are rapidly cleared from the blood circulation, and therefore have low tumor penetration. Historically, nanocarrier localization in tumors was believed to be due to the enhanced permeability and retention (EPR) effect. However, more recently the EPR concept has come under increasing criticism and some nanomedicine scientists postulate that the EPR effect exists only in mouse tumour models and not in humans[15]. A simple solution to achieve maximum tumor accumulation by passive targeting is to engineer nanocarriers with antifouling surfaces, that can avoid nonspecific serum-protein adsorption and prolong circulation time[16]. The low EPR effect and the heterogeneity of tumors can be partially overcome by designing HNPs with stealth coatings, and large targetted payloads of functional agents. One option would be to use a targeted RBC-membrane-enveloped nanosponge (RBC@NS) [17]. Under near infrared (NIR) light the nanosponge acts as a photopenetrative and photolytic agent which further damages deep tumor tissues by the localized heat energy.

The ability to control and monitor chemotherapeutic drug release from HNPs is an important property for effective and powerful cancer theranostics. Monitoring drug release could help cancer specialists and clinicians to control surplus chemotherapeutic-induced side effects or inadequate dosages. Furthermore, controlled drug release and monitoring may allow for clinical decisions to be made for patient-specific personalized medicine approach. HNPs have been developed which monitor real-time quantitative drug release profiles using radio imaging modalities such as magnetic resonance imaging (MRI)[18]. In principle, the HNPs are loaded with chemotherapeutic drugs, and with radio imaging agents to directly image and quantify tumor accumulation. Such a platform provides very specific information on (i) biodistribution and tumor accumulation; (ii) premature drug release from the surface (iii) real-time monitoring of drug release in the the tumor. 
Combining drug delivery with thermal therapies such as magnetic hyperthermia (MH), photothermal therapy (PTT) and photodynamic therapy (PDT) has the potential for more effective cancer treatment $[19,20]$. The duplex effect of local heating can controlthe amount of drug released, and also spatial control of the release. Furthemore, the elevated temperature can increasethe effectiveness of the drug. Inspired by these interesting dual modalities of HNPs, preclinical reports have shownefficient photothermal damage of primary tumors under MRIphotoacoustic (PA) imaging guidance, and hyperthermia-mediated immunostimulatory effect on deep seated tumors[21] (Figure 1). In another approach, a reactive oxygen species (ROS)responsive core-cross-linked HNP micellar system has been designed to enhance the circulation stability and to allow on-demand chemotherapeutic drug release via far red light induced ROS generation upon [22]. Noninvasive magnetic fields in conjunction with hybrid superparamagnetic nanoparticles offers spatial and temporal controlled stimuli-responsive drug delivery [23]. Interestingly, HNPs based cancer theranostics not only provide theranostic agent to assist tumor imaging and therapy, but also give an avenue to develop theranostic agent combined MRI, chemotherapy, and PTT in single platform to combat cancer in advanced stage[24,25]. As an alternative to companion radio imaging and thermal therapy, NIR-II imaging combined with the noninvasive focused ultrasound (FUS) is developed to detect and treat the orthotopic brain tumor[26]. The designed HNP allowing intravenously injected nanocarriers to passively penetrate into brain through the blood-brain-barrier (BBB) that was temporarily opened by the FUS treatment. Such a novel HNP demonstrated a potential application to enable easy and efficient in vivo monitoring of target site accumulation and theranostics. This smart and straightforward approach to combat cancer opens up new theranostic opportunities for patient stratification in cancer nanomedicine. 


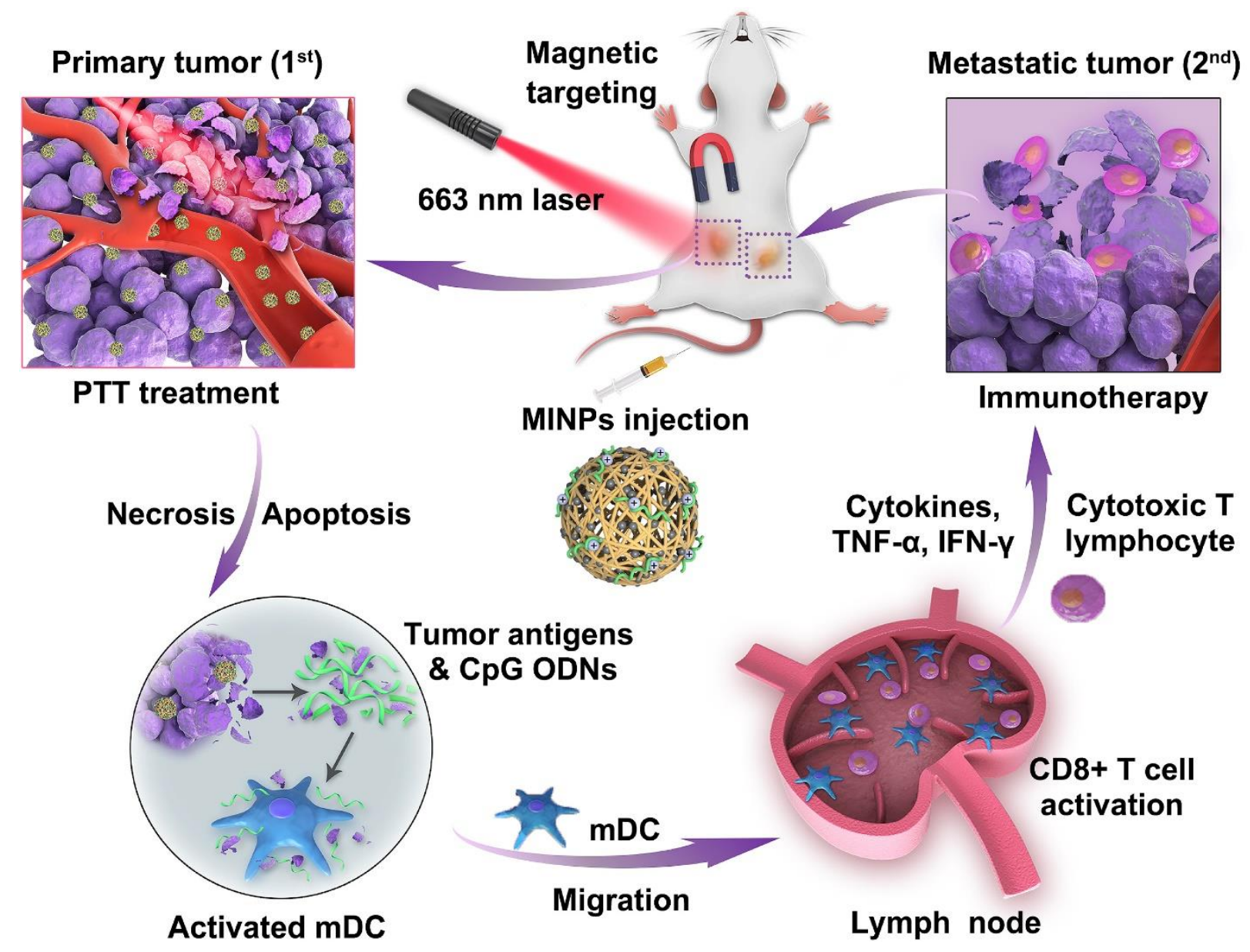

Figure 1. Schematic illustration of the imaging-guided photothermally triggered immunotherapy based on the magnetic-responsive immunostimulatory nanoagents (MINPs) for both primary treated and distant untreated tumors[21].

The last two decades witnessed the development of first-generation nanotheranostics that simplified the design, size, shape and functionality of nanostructures. Simplified in their design, these nanostructures were regarded as vectors for drug delivery that improved tumor localization, blood circulation time and pharmacokinetics[27]. Although they proved beneficial in terms of therapy, drug delivery and reducing drug-associated toxicity, they have yet to translate to an advanced clinical stage to achieve significantly improved patient outcomes. Next generation HNPs are moving beyond the confines of convention in terms of size, shape, surface functionality, and in vitro toxicity. The technology is transitioning towards more rational approaches of HNPs that take into consideration advanced clinical settings and biological barriers[28]., Hybrid nanorobot 
systems, for example, have been designed to incoporateMR and CT imaging contrast agent, chemotherapeutic cargo, tumor targeting, and light absorbing molecules. This is designed to monitor nanocarrierbiodistribution, tumor size, and tumor therapeutic efficacy[29]. Another promising strategy to overcome biological barriers and enhance cancer therapeutic efficacy is to use mechanism-based enhancement of cancer PDT using biomimetic metal-organic framework (MOF) nanoplatforms[30]. This HNP was used to deliver the antiangiogenic drug apatinib, and showed excellent synergistic effects in combination with MOF-based PDT. Thisapproach inhibits the protumoral post-PDT angiogenesis and offers a precise design to compact solutions for multiple obstacles in cancer theranostics using a single formulation. Numerous research activities and resources are continually directed in the designing of innovative nanotheranostics using HNPs for proper real time tumor imaging tools to assess the progression of diseases and their therapeutic response[31,32]. Often times, these HNPs include lanthanide-based excretable NIR-II active nanostructures in the second near-infrared window for tumor imaging and surgical navigation [33,34] (Figure 2) or PTT-responsive hybrid nano polymersomes. These have been shown to be versatile therapeutics for effective breast tumor suppression[35].

With improved understanding of the molecular biology of tumors, HNPs can be rationally designed to enhance the accessibility of chemotherapeutic drugs to cancer tissues. At present, HNPs are more focused on delivering highly cytotoxic chemo-drug and their release in the tumor micro milieu to selective kill cancer cells by cellular apoptosis or necrosis[36]. While, this selective therapy is advantageous it has limited penetration due to the complex outer structure of the tumor and its microenvironment. Using new generation HNPs this can be further resolved by originating, catalyzing or triggering specific chemical reactions once inside a tumor, which can create localized special chemicals and products to initiate a series of unique biological and pathologic effects. Tumor-specific enzymatic reactions, for example, could prolong drug exposure and form intracellular nano-assemblies to monitor the cancer therapy [37]. A similar strategy of tumor-specific intracellular HNPs uses a bioinspired lipoprotein (bLP) assembly which prompts anticancer drug accessibility to tumor cells and induces efficient photothermia for antitumor therapy [38]. Several other pragmatic research studies have proposed next generation HNPs to treat primary and metastatic breast cancer[39] and drug resistance colon cancer[40]. Such approaches can be expected to increase the localization of anticancer drugs, and increase sensitivity, while reducing off-target toxicity. 


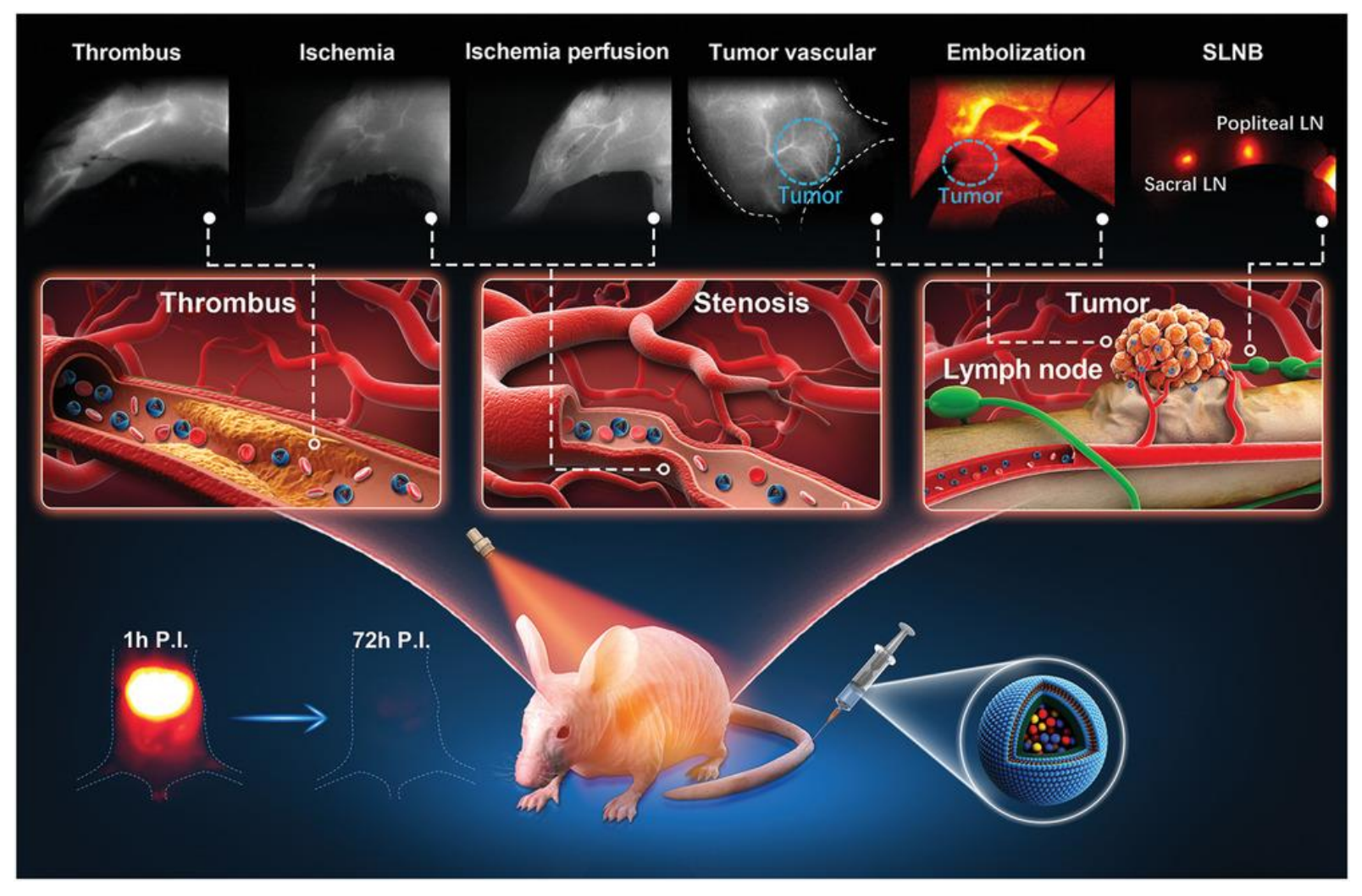

Figure 2. Schematic illustration of next generation HNP which consists with excretable lanthanide nanoparticle for multifunctional biomedical imaging and surgical navigation in the second near-infrared window. The strategy reports innovative nanotheranostics using HNPs for proper real time tumor imaging tools to assess the progression of diseases and their therapeutic response[34].

\section{Hybrid nanoplatforms: Clinical translation}

In the ever-continuing arms race between cancer and the specialists tackling it, nanotheranostics has emerged as a useful ally. However, the significant preclinical anticancer activity demonstrated by HNCs has yet to be recaptured in a clinical setting, and, as a result, the development of the marketed nanomedicines has often been slow. The rapid transformation of HNPs from the laboratory scale to advanced clinical stage requires in depth pathological and pharmacological evaluation. To be effective the HNPs must first and foremost be capable of reaching the disease site or site of the lesion[41]. Several experimental studies have established 
the conclusion that in vivo chemotherapeutic drug delivery is a complex process and difficult to predict the relationship between in vitro and in vivo delivery[42,43].

\title{
Table: 1 HNPs in clinical stage: considerations and development
}

\author{
HNPs surface design \\ Key Considerations \\ - Optimized particle surface size \\ - Grafting targeting agents, and anticancer drugs in a single modality \\ - Biocompatibility and biodegradability \\ - Achieve the tumor targeting and tumor localization, only \\ - Stimulated/triggered drug release in the tumor microenvironment \\ Advances \\ - HNPs inherent physical and chemical properties (e.g., magnetic, light, ultrasound, pH \\ responsiveness) upon stimulation are beneficial for remote controlled therapies \\ - HNPs unique structure allow multiple chemotherapeutic cargoes within a single structure, which \\ is supporting the codelivery of multiple cargoes to enhance therapeutic efficacy and overcome \\ cancer cells drug resistance
}

\section{Current limitations}

- Large-scale production, reproducibility, implementing the GMP standards

- Unknown cytotoxicity profiles

\section{HNPs preclinical evolution}

Key Considerations

- Intracellular trafficking, bio-interface, in vivo toxicity and degradation

- Visualization and monitoring of tumor localized drug delivery

- Active and passive tumor targeting

- Transportation of nanocarriers across tight epithelial and endothelial barriers such as the BBB

Advances

- Enhancement of the pharmacokinetics including stability, solubility and blood circulation

- Enhancement of the tumor penetration and drug accumulation

- Enhancement of the cellular uptake and intracellular trafficking

Current limitations

- Problems of reproducibility and the major biases raised due to diverse types of preclinical research

- Tumor microenvironment navigation to nanocarriers

- Avoiding nonspecific interactions between nanocarriers and serum proteins

- Monitoring the real-time feedback on the in vivo anticancer efficacy of a therapeutic cargo

\section{HNPs clinical evolution}

\section{Key Considerations}

- Advanced imaging modalities 'seeing is believing'

- Chemotherapeutic cargo delivery to the major sites of metastasis (e.g., lungs, liver, lymph nodes, brain, and bone)

- Selective treatment imaging guided focal therapy by nanotheranostics

- Adjunct therapeutic approach

Advances

- HNPs combined with radio imaging modalities (MRI, CT, PET.etc) support real-time therapy monitoring or treatment feedback 
- HNPs are beneficial over conventional therapies in miniaturized medical devices for diagnosis, drug screening, and delivery

\section{Current limitations}

- Clinical studies only on induced clinical models

- Tumor heterogeneity and patient stratification

- Limited understanding of the human physiology and organ interaction with the nanocarriers

- High costs of materials and advanced stage clinical protocols

\section{Commercialization}

\section{Key Considerations}

- Generalization of advanced invention to commercialization pathways

- Interdisciplinary approach with diversity in expertise

- Enhancing trust of the research by collaboration between investigators and biomedical scientists

- Sharing the research data, animal protocols, designed materials, developed software's and code

\section{Advances}

- Controllable and reproducible platforms and scalable manufacturing of HNPs

- Optimal dose screening with risk assessment is possible

- Skilled and experienced human resource is available

- Data transparency and sharing of resources is possible

\section{Current limitations}

- Researchers focus on publications rather than clinical translation

- Lack of clear regulatory guidelines by different authorities specific for nanomedicine

- Undefined and complex patents and IP right guidelines

- Difference in mindset or intellectual culture between nanotechnologists, doctors, oncologists and investors

- Investors mindset for success and the risk of failure with minimal initial investment 


\section{Hybrid Nanoplatforms (HNPs)}
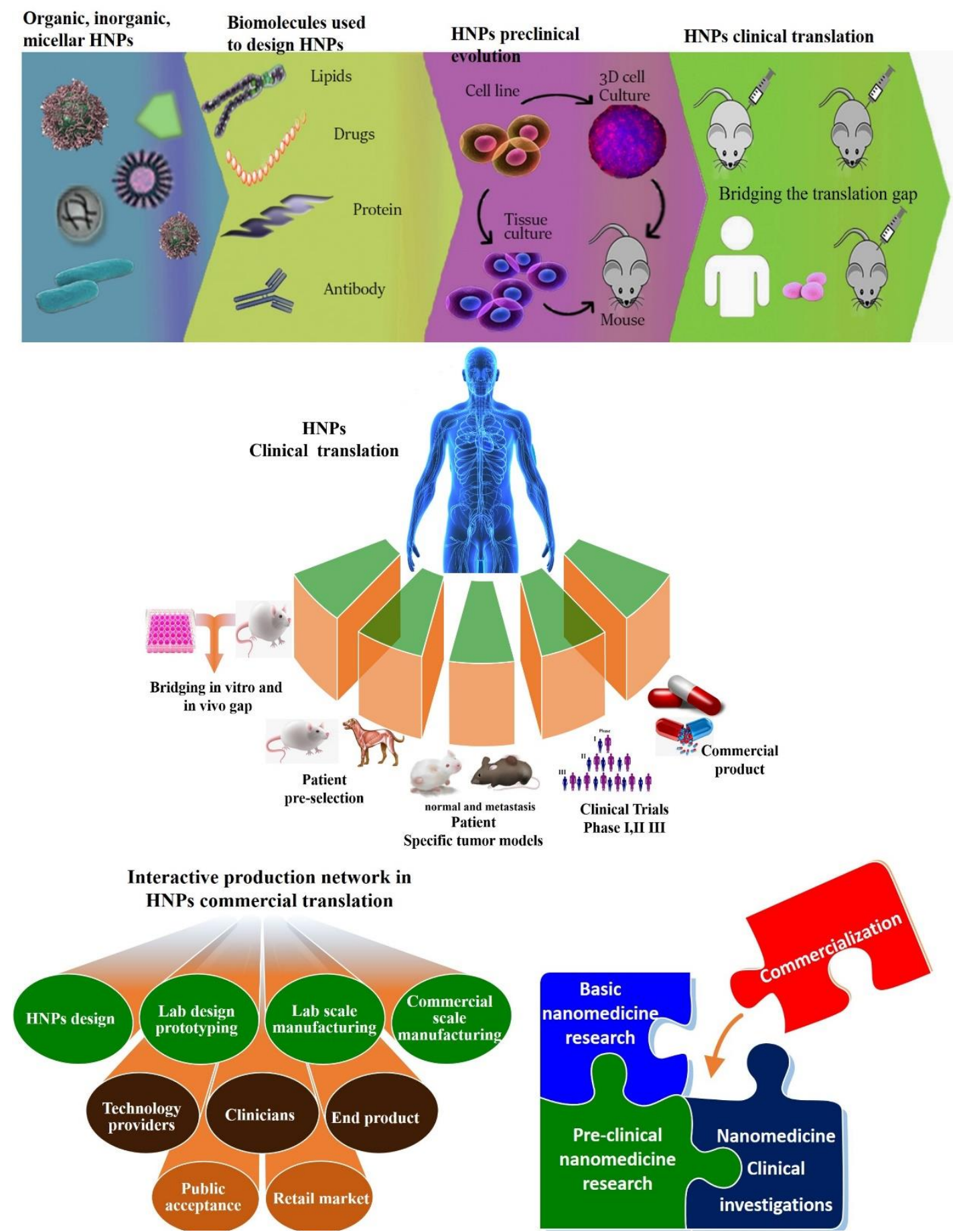

Figure 3. Overview of approaches for HNPs development for cancer theranostics. HNPs design, clinical stage transformation, considerations and development processes. HNPs translation to clinic face challenges in controlling the physio-chemical properties, interactions with specific biological systems and the animal models used to characterize in clinical end points. The final commercialization of HNPs or overall nanomedicine involves different stakeholders. 


\section{Conclusion and Future perspectives}

Cancer is a heterogeneous disease associated with distinct cell subpopulations. Understanding tumor biology is critical for the development of successful theranostic applications since cancer cells have different capacities to grow, differentiate, develop drug resistance, and form metastases[44]. A deep understanding between cancer cells and theranostic nanomaterials must be accomplished considering a biological perspective[45]. Thus, great efforts have been directed by the scientific research community to translate these new theranostics nanoplatforms into clinical trials.

Specific drug delivery to a target tumor site is one of the greatest challenges in cancer theranostics. Although current theranostics nanomaterials have great potential, next-generation design concepts and their effective implementation strategies are required. Many different nanoplatforms have been evaluated for drug targeting to tumors, several of them have managed to get FDA approval and are routinely used in the clinic. The majority, however, have failed either in late-stage preclinical or in early-stage clinical trials. In the future efforts, it will be important to not only make ever more nanomaterials, but also to understand sub-cellular level interactions between cancer cells and nanomaterials. It is necessary to understand the mechanisms of the interactions of targeted theranostics nanomaterial with tumor cells and the tumor microenvironment. In addition, future nanosystems should be able to pass through biological barriers to reach any tumor sites of the body[46-48].

The main aim of efficient nanoplatforms is to reduce the drug dose needed to achieve a specific therapeutic effect, thus lowering the costs and reducing the side effects associated with their use. Organic nanocarriers have properties better matched to the physicochemical conditions encountered in biological tissues, thus furnishing the best examples of biocompatible nanoplatforms. On the other hand, inorganic materials possess complementary functions for the diagnosis and detection of the pathological conditions within the diseased tissues[49].

The main challenge in the preclinical characterization is the need for the complete understanding of the nanoformulations. A multidisciplinary team of experts is required for an advanced evaluation of a nanomedicine, especially at the preclinical stage. A large variety of data is now available at the preclinical level of analysis for different nanosystems; however, it will be difficult to draw firm conclusions from their results unless studies are systematically planned due to variations in cell lines and animal models. So, one can predict, with such approaches for a 
nanoformulation, in vivo results and determine whether the in vivo experiments are warranted or need to be redesigned. In vitro studies for immune response are largely complete and now further analysis of the in vivo immunological response of nanoformulations is needed.

One outstanding issue concerns the reproducibility of nanomedicine formulations. Formulation of homogeneous nanomedicines is still a challenge. Another subject of interest is the stability of formulations under the various conditions that might approximate its use. Scrutiny is therefore needed at every stage of the production process. Ideal nanomedicines will have a modular design that can be easily scaled up for Good Manufacturing Practices and can be stored for a long time prior to use in patients[50]. Incorporation of multiple drugs, and imaging agents, within HNCs on a large scale might be expensive as well as challenging. Further studies and optimizations will be required to eventually scale up the manufacturing process from the bench to clinical application [51]. The GMP manufactured theranostics nanomedicine should be simple, cheap, ultra-small, biodegradable, and easy to use. In addition, they should possess high imaging signal strengths, high drug loading capacity, and controlled drug release ability.

In conclusion, significant research is still required to understand and predict how these materials will behave in biological systems, and how to undertake large-scale production of GMP grade theranostic nanoparticles for human use. We hope that in the coming years many nanoplatforms will get FDA approval and will be in routine use in clinics to improve cancer patient survival.

\section{ACKNOWLEDGMENTS}

The project leading to this research has received funding from the European Union's Horizon 2020

research and innovation programme under the Marie Sklodowska-Curie Grant Agreement No. 751903.

\section{References:}

[1] Bray LJ, Hutmacher DW, Bock N. Addressing Patient Specificity in the Engineering of Tumor Models. Front Bioeng Biotechnol 2019;7. https://doi.org/10.3389/fbioe.2019.00217.

[2] Yu G, Cen T-Y, He Z, Wang S-P, Wang Z, Ying X-W, et al. Porphyrin Nanocage- 
Embedded Single-Molecular Nanoparticles for Cancer Nanotheranostics. Angew Chemie Int Ed 2019;58:8799-803. https://doi.org/10.1002/anie.201903277.

[3] Boehnke N, Correa S, Hao L, Wang W, Straehla JP, Bhatia SN, et al. Theranostic Layerby-Layer Nanoparticles for Simultaneous Tumor Detection and Gene Silencing. Angew Chemie - Int Ed 2020. https://doi.org/10.1002/anie.201911762.

[4] Tang W, Fan W, Lau J, Deng L, Shen Z, Chen X. Emerging blood-brain-barrier-crossing nanotechnology for brain cancer theranostics. Chem Soc Rev 2019;48:2967-3014. https://doi.org/10.1039/c8cs00805a.

[5] Liao J, Jia Y, Wu Y, Shi K, Yang D, Li P, et al. Physical-, chemical-, and biologicalresponsive nanomedicine for cancer therapy. Wiley Interdiscip Rev Nanomedicine Nanobiotechnology 2020;12. https://doi.org/10.1002/wnan.1581.

[6] Ioannidis JPA, Kim BYS, Trounson A. How to design preclinical studies in nanomedicine and cell therapy to maximize the prospects of clinical translation. Nat Biomed Eng 2018;2:797-809. https://doi.org/10.1038/s41551-018-0314-y.

[7] Peng J, Yang Q, Shi K, Xiao Y, Wei X, Qian Z. Intratumoral fate of functional nanoparticles in response to microenvironment factor: Implications on cancer diagnosis and therapy. Adv Drug Deliv Rev 2019;143:37-67. https://doi.org/10.1016/j.addr.2019.06.007.

[8] Guo P, Yang J, Liu D, Huang L, Fell G, Huang J, et al. Dual complementary liposomes inhibit triple-negative breast tumor progression and metastasis. Sci Adv 2019;5. https://doi.org/10.1126/sciadv.aav5010.

[9] Thorat ND, Townely H, Brennan G, Parchur AK, Silien C, Bauer J, et al. Progress in Remotely Triggered Hybrid Nanostructures for Next-Generation Brain Cancer Theranostics. ACS Biomater Sci Eng 2019;5:2669-87. https://doi.org/10.1021/acsbiomaterials.8b01173.

[10] An HW, Li LL, Wang Y, Wang Z, Hou D, Lin YX, et al. A tumour-selective cascade activatable self-detained system for drug delivery and cancer imaging. Nat Commun 2019;10:4861. https://doi.org/10.1038/s41467-019-12848-5.

[11] Wang S, Huang P, Chen X. Stimuli-Responsive Programmed Specific Targeting in Nanomedicine. ACS Nano 2016;10:2991-4. https://doi.org/10.1021/acsnano.6b00870.

[12] Thorat ND, Otari SV, Patil RM, Khot VM, Prasad AI, Ningthoujam RS, et al. Enhanced colloidal stability of polymer coated $\mathrm{La} 0.7 \mathrm{Sr} 0.3 \mathrm{MnO} 3$ nanoparticles in physiological media for hyperthermia application. Colloids Surfaces B Biointerfaces 2013;111. https://doi.org/10.1016/j.colsurfb.2013.06.014.

[13] Tran S, DeGiovanni P-J, Piel B, Rai P. Cancer nanomedicine: a review of recent success in drug delivery. Clin Transl Med 2017;6:44. https://doi.org/10.1186/s40169-017-0175-0.

[14] Kevadiya BD, Ottemann BM, Thomas M Ben, Mukadam I, Nigam S, McMillan JE, et al. Neurotheranostics as personalized medicines. Adv Drug Deliv Rev 2019;148:252-89. https://doi.org/10.1016/j.addr.2018.10.011.

[15] van der Meel R, Sulheim E, Shi Y, Kiessling F, Mulder WJM, Lammers T. Smart cancer nanomedicine. Nat Nanotechnol 2019;14:1007-17. https://doi.org/10.1038/s41565-0190567-y.

[16] Van Haute D, Liu AT, Berlin JM. Coating Metal Nanoparticle Surfaces with Small Organic Molecules Can Reduce Nonspecific Cell Uptake. ACS Nano 2018;12:117-27. https://doi.org/10.1021/acsnano.7b03025.

[17] Sung S-Y, Su Y-L, Cheng W, Hu P-F, Chiang C-S, Chen W-T, et al. Graphene Quantum 
Dots-Mediated Theranostic Penetrative Delivery of Drug and Photolytics in Deep Tumors by Targeted Biomimetic Nanosponges. Nano Lett 2019;19:69-81.

https://doi.org/10.1021/acs.nanolett.8b03249.

[18] Ding X, Zhao H, Li C, Wang Q, Jiang J. All-in-one theranostic nanoplatform with controlled drug release and activated MRI tracking functions for synergistic NIR-II hyperthermia-chemotherapy of tumors. Nano Res 2019. https://doi.org/10.1007/s12274019-2540-3.

[19] Alphandéry E. Iron oxide nanoparticles for therapeutic applications. Drug Discov Today 2019:141-9. https://doi.org/10.1016/j.drudis.2019.09.020.

[20] Thorat ND, Tofail SAM, Von Rechenberg B, Townley H, Brennan G, Silien C, et al. Physically stimulated nanotheranostics for next generation cancer therapy: Focus on magnetic and light stimulations. Appl Phys Rev 2019;6. https://doi.org/10.1063/1.5049467.

[21] Guo Y, Ran Y, Wang Z, Cheng J, Cao Y, Yang C, et al. Magnetic-responsive and targeted cancer nanotheranostics by PA/MR bimodal imaging-guided photothermally triggered immunotherapy. Biomaterials 2019;219:119370. https://doi.org/10.1016/j.biomaterials.2019.119370.

[22] Li Y, Hu J, Liu X, Liu Y, Lv S, Dang J, et al. Photodynamic therapy-triggered on-demand drug release from ROS-responsive core-cross-linked micelles toward synergistic anticancer treatment. Nano Res 2019;12:999-1008. https://doi.org/10.1007/s12274-019-2330$\mathrm{y}$.

[23] Chen W, Cheng CA, Zink JI. Spatial, Temporal, and Dose Control of Drug Delivery using Noninvasive Magnetic Stimulation. ACS Nano 2019;13:1292-308. https://doi.org/10.1021/acsnano.8b06655.

[24] Zhao Z, Xu K, Fu C, Liu H, Lei M, Bao J, et al. Interfacial engineered gadolinium oxide nanoparticles for magnetic resonance imaging guided microenvironment-mediated synergetic chemodynamic/photothermal therapy. Biomaterials 2019;219:119379. https://doi.org/10.1016/j.biomaterials.2019.119379.

[25] Zhang C, Li J, Yang C, Gong S, Jiang H, Sun M, et al. A pH-sensitive coordination polymer network-based nanoplatform for magnetic resonance imaging-guided cancer chemo-photothermal synergistic therapy. Nanomedicine Nanotechnology, Biol Med 2020;23:102071. https://doi.org/10.1016/j.nano.2019.102071.

[26] Liu Z, Ren F, Zhang H, Yuan Q, Jiang Z, Liu H, et al. Boosting often overlooked long wavelength emissions of rare-earth nanoparticles for NIR-II fluorescence imaging of orthotopic glioblastoma. Biomaterials 2019;219:119364. https://doi.org/10.1016/j.biomaterials.2019.119364.

[27] Blanco E, Shen H, Ferrari M. Principles of nanoparticle design for overcoming biological barriers to drug delivery. Nat Biotechnol 2015;33:941-51. https://doi.org/10.1038/nbt.3330.

[28] Patil RM, Thorat ND, Shete PB, Otari S V., Tiwale BM, Pawar SH. In vitro hyperthermia with improved colloidal stability and enhanced SAR of magnetic core/shell nanostructures. Mater Sci Eng C 2016;59:702-9. https://doi.org/10.1016/j.msec.2015.10.064.

[29] Jin Z, Nguyen KT, Go G, Kang B, Min H-K, Kim S-J, et al. Multifunctional Nanorobot System for Active Therapeutic Delivery and Synergistic Chemo-photothermal Therapy. Nano Lett 2019;19:8550-64. https://doi.org/10.1021/acs.nanolett.9b03051. 
[30] Min H, Wang J, Qi Y, Zhang Y, Han X, Xu Y, et al. Biomimetic Metal-Organic Framework Nanoparticles for Cooperative Combination of Antiangiogenesis and Photodynamic Therapy for Enhanced Efficacy. Adv Mater 2019;31:1808200. https://doi.org/10.1002/adma.201808200.

[31] Zhang Q, Zhou H, Chen H, Zhang X, He S, Ma L, et al. Hierarchically Nanostructured Hybrid Platform for Tumor Delineation and Image-Guided Surgery via NIR-II Fluorescence and PET Bimodal Imaging. Small 2019;15:1903382. https://doi.org/10.1002/smll.201903382.

[32] Zhang X, He S, Ding B, Qu C, Zhang Q, Chen H, et al. Cancer cell membrane-coated rare earth doped nanoparticles for tumor surgery navigation in NIR-II imaging window. Chem Eng J 2020;385:123959. https://doi.org/10.1016/j.cej.2019.123959.

[33] Liu Y, Liu J, Chen D, Wang X, Liu Z, Liu H, et al. Quinoxaline-Based Semiconducting Polymer Dots for in Vivo NIR-II Fluorescence Imaging. Macromolecules 2019;52:573540. https://doi.org/10.1021/acs.macromol.9b01142.

[34] Li D, He S, Wu Y, Liu J, Liu Q, Chang B, et al. Excretable Lanthanide Nanoparticle for Biomedical Imaging and Surgical Navigation in the Second Near-Infrared Window. Adv Sci 2019;6:1902042. https://doi.org/10.1002/advs.201902042.

[35] Zhang H, Cui W, Qu X, Wu H, Qu L, Zhang X, et al. Photothermal-responsive nanosized hybrid polymersome as versatile therapeutics codelivery nanovehicle for effective tumor suppression. Proc Natl Acad Sci U S A 2019;116:7744-9. https://doi.org/10.1073/pnas.1817251116.

[36] Thorat ND, Brennan G, Bauer J, Silien C, Tofail SAM. Strengths and Limitations of Translating the Hybrid Nanostructures to the Clinic. Hybrid Nanostructures for Cancer Theranostics, Elsevier; 2019, p. 229-54. https://doi.org/10.1016/b978-0-12-8139066.00012-3.

[37] Yuan Y, Zhang J, Qi X, Li S, Liu G, Siddhanta S, et al. Furin-mediated intracellular selfassembly of olsalazine nanoparticles for enhanced magnetic resonance imaging and tumour therapy. Nat Mater 2019. https://doi.org/10.1038/s41563-019-0503-4.

[38] Tan T, Hu H, Wang H, Li J, Wang Z, Wang J, et al. Bioinspired lipoproteins-mediated photothermia remodels tumor stroma to improve cancer cell accessibility of second nanoparticles. Nat Commun 2019;10:3322. https://doi.org/10.1038/s41467-019-11235-4.

[39] Dancy JG, Wadajkar AS, Connolly NP, Galisteo R, Ames HM, Peng S, et al. Decreased nonspecific adhesivity, receptor-targeted therapeutic nanoparticles for primary and metastatic breast cancer. Sci Adv 2020;6:eaax3931. https://doi.org/10.1126/sciadv.aax3931.

[40] Thorat ND, Bauer J, Tofail SAM, Gascón Pérez V, Bohara RA, Yadav HM. Silica nano supra-assembly for the targeted delivery of therapeutic cargo to overcome chemoresistance in cancer. Colloids Surfaces B Biointerfaces 2020;185. https://doi.org/10.1016/j.colsurfb.2019.110571.

[41] Hua S, de Matos MBC, Metselaar JM, Storm G. Current trends and challenges in the clinical translation of nanoparticulate nanomedicines: Pathways for translational development and commercialization. Front Pharmacol 2018;9. https://doi.org/10.3389/fphar.2018.00790.

[42] Paunovska K, Sago CD, Monaco CM, Hudson WH, Castro MG, Rudoltz TG, et al. A Direct Comparison of in Vitro and in Vivo Nucleic Acid Delivery Mediated by Hundreds of Nanoparticles Reveals a Weak Correlation. Nano Lett 2018;18:2148-57. 
https://doi.org/10.1021/acs.nanolett.8b00432.

[43] Paunovska K, Gil CJ, Lokugamage MP, Sago CD, Sato M, Lando GN, et al. Analyzing 2000 in Vivo Drug Delivery Data Points Reveals Cholesterol Structure Impacts

Nanoparticle Delivery. ACS Nano 2018;12:8341-9.

https://doi.org/10.1021/acsnano.8b03640.

[44] Thorat ND, Bauer J, Tofail SAM, Gascón Pérez V, Bohara RA, Yadav HM. Silica nano supra-assembly for the targeted delivery of therapeutic cargo to overcome chemoresistance in cancer. Colloids Surfaces B Biointerfaces 2020;185:110571. https://doi.org/10.1016/j.colsurfb.2019.110571.

[45] Silva CO, Pinho JO, Lopes JM, Almeida AJ, Gaspar MM, Reis C. Current Trends in Cancer Nanotheranostics: Metallic, Polymeric, and Lipid-Based Systems. Pharmaceutics 2019;11:22. https://doi.org/10.3390/pharmaceutics11010022.

[46] Pershina AG, Brikunova OY, Demin AM, Shevelev OB, Razumov IA, Zavjalov EL, et al. pH-triggered delivery of magnetic nanoparticles depends on tumor volume. Nanomedicine Nanotechnology, Biol Med 2020;23:102086. https://doi.org/10.1016/j.nano.2019.102086.

[47] Lammers T, Kiessling F, Hennink WE, Storm G. Drug targeting to tumors: Principles, pitfalls and (pre-) clinical progress. J Control Release 2012;161:175-87. https://doi.org/10.1016/j.jconrel.2011.09.063.

[48] Thorat ND. External Field and Radiation Stimulated Breast Cancer Nanotheranostics. IOP Publishing; 2019. https://doi.org/10.1088/2053-2563/ab2907.

[49] Lombardo D, Kiselev MA, Caccamo MT. Smart Nanoparticles for Drug Delivery Application: Development of Versatile Nanocarrier Platforms in Biotechnology and Nanomedicine. J Nanomater 2019;2019:26. https://doi.org/10.1155/2019/3702518.

[50] Tran S, DeGiovanni P-J, Piel B, Rai P. Cancer nanomedicine: a review of recent success in drug delivery. Clin Transl Med 2017;6. https://doi.org/10.1186/s40169-017-0175-0.

[51] Hameed S, Bhattarai P, Dai Z. Cerasomes and bicelles: Hybrid bilayered nanostructures with silica-like surface in cancer theranostics. Front Chem 2018;6. https://doi.org/10.3389/fchem.2018.00127. 